\title{
Research Paper \\ Antibacterial Activity and Antibiofilm Properties of Satureja Essential Oil Against Periodontal Pathogens
}

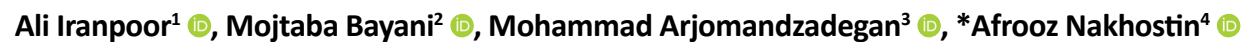

1. Dentist, Arak, Iran.

2. Department of Periodontics, School of Dentistry, Arak University of Medical Sciences, Arak, Iran.

3. Tuberculosis and Pediatrics Infectious Diseases Research Center, Department of Microbiology and Immunology, School of Medicine, Arak University of Medical Sciences, Arak, Iran.

4. Department of Restorative and Aesthetic Dentistry, School of Dentistry, Arak University of Medical Sciences, Arak, Iran.

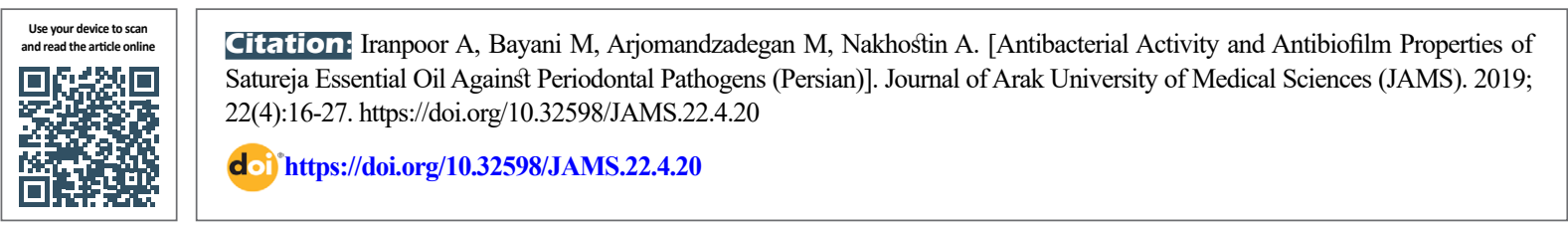

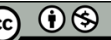

Article Info:

Received: 20 May 2019 Accepted: 25 Aug 2019 Available Online: 01 Oct 2019

Keywords: Periodontal pathogens, Satureja plant, Antibacterial activity Antibiofilm

\section{A B STRACT}

Background and Aim Periodontal diseases are among the most prevalent inflammatory diseases caused by oral bacteria. Expansion of oral biofilm causes various diseases such as gingival inflammation and periodontitis. The Satureja plant has various species, all of which are aromatic. This plant is traditionally used for the treatment of some diseases. The present study was conducted to evaluate the effect of Satureja essential oil on periodontal pathogens.

Methods and Materials In this study, we evaluated four pathogens; Enterococcus faecalis, Streptococcus sanguinis, Eikenella corrodens, and Actinomyces viscosus. We also used the disk diffusion test and broth microdilution method to evaluate the antimicrobial effect of Satureja essential oil. Finally, we determined the minimum inhibitory concentration and minimum bactericidal concentration.

Ethical Considerations The Ethics Committee of Arak University of Medical Sciences approved this study (Code: IR.ARAKMU.REC.1397.67).

Results In concentration of $0.1 \mathrm{~g} / \mathrm{mL}$ of Satureja plant, Actinomyces viscosus, Streptococcus sanguinis, Enterococcus faecalis, and Eikenella corrodens were found to be sensible to resistance. The Satureja essential oil had the highest effect on Eikenella corrodens. Results obtained from the biofilm test showed no biofilm in a concentration of $12.5 \mathrm{mg} / \mathrm{mL}$ and higher of Satureja plant.

Conclusion The Satureja plant was found to have an antibacterial and inhibitory effect on biofilm growth and formation in the oral cavity.

\section{Extended Abstract}

\section{Introduction}

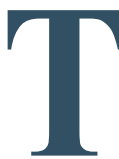

ooth decay and periodontal disease are the most common infectious human diseases that are caused by oral bacteria such as Staphylococcus aureus, Micrococcus, Pseudomonas,
Candida, etc. [1]. Various ways, such as chemical mouthwashes, toothpaste, and antibiotics, are used to prevent and treat periodontal disease and tooth decay $[10,11,13]$.

Herbal and other natural antibacterial materials are now considered as useful and alternative antibacterial methods for oral and toothpaste washing [14]. Satureja has numerous compounds with antibacterial properties [15].

\section{* Corresponding Author:}

\section{Afrooz Nakhostin, MD.}

Address: Department of Restorative and Aesthetic Dentistry, School of Dentistry, Arak University of Medical Sciences, Arak, Iran

Tel: +98 (918) 3602208

E-mail: afr_na_sa@yahoo.com 
Since the oral environment contains many bacterial species and the use of chemical drugs in the oral cavity is associated with complications such as changes in the natural flora, in this study, we investigated the effect of Satureja oil on some common oral bacteria.

\section{Materials and Methods}

This experimental study was performed on microbial strains provided from Iran Microbial Bank (including Enterococcus faecalis, Streptococcus sanguis, Eikenella corrodens, and Actinomyces viscose). Some fresh Satureja was obtained from the Research Center for Medicinal Plants of Shahid Beheshti University, and its essential oil by water distillation or Clevenger apparatus.

The disk diffusion method was used to determine microbial susceptibility to essential oil after preparation of microbial suspension at half McFarland concentration and its culturing as aqueous media on Muller-Hinton Agar medium. The blank disk was immersed in $20 \mu \mathrm{L}$ of essential oil with concentrations of $1 \mathrm{~g} / \mathrm{mL}$ and $0.1 \mathrm{~g} / \mathrm{mL}$ on the culture medium and placed at a suitable distance from the plate incubated at $37^{\circ} \mathrm{C}$ for 48 hours. After the incubation period, the bacterial growth inhibition zone diameter was measured in millimeters. The experiment was repeated 3 times, and the mean results was reported.

The microplate dilution method was also used to determine the antimicrobial effect of the essential oil. Median inhibitory and lethal concentrations were also determined in this study. The microplate method was also used to evaluate the effect of essential oil on biofilm formation inhibition.

\section{Results}

The results of microbial susceptibility to Satureja essential oil showed that the highest diameter of $30 \mathrm{~mm}$ growth inhibition zone was related to the effect of Satureja essential oil at a concentration of $1 \mathrm{~g} / \mathrm{mL}$ on the growth of Streptococcus sanguis and Eikenella corrodens. The lowest diameter of 9 mm growth inhibition zone was due to the effect of essential oil of $0.1 \mathrm{~g} / \mathrm{mL}$ on the growth of Eikenella corrodens. Table 1 presents the results of the evaluation of the median inhibitory concentration and median lethal concentration of the essential oil of the Satureja on the studied bacteria.

The median growth inhibitory concentration and the median growth inhibitory concentration were presented in $\mu \mathrm{g} / \mathrm{mL}$. The results of the microplate dilution test showed that the studied bacteria at higher concentrations of $1.562 \mathrm{mg} / \mathrm{mL}$ of Satureja essential oil lost their growth ability but still retain their growth potential at lower concentrations of $0.195 \mathrm{mg}$ / $\mathrm{mL}$ of this essential oil.

The results of the biofilm inhibition test showed that the studied bacteria did not form biofilms at concentrations higher than or equal to $12.5 \mathrm{mg} / \mathrm{mL}$, but the biofilms were formed at concentrations below $0.39 \mathrm{mg} / \mathrm{mL}$.

\section{Discussion}

The present study was carried out to investigate the antibacterial activity of the essential oil of Satureja by disk diffusion and microplate dilution methods. The microplate technique was also used to examine the effect of essential oil on biofilm formation. The results of the disk diffusion method, considering the size of the bacterial growth inhibition zone, showed that pure Satureja essential oil $(1 \mathrm{~g} / \mathrm{mL})$ had a strong antimicrobial activity compared to essential oil at $0.1 \mathrm{~g} / \mathrm{mL}$.

By examining the results of the microplate dilution test, the median concentration of essential oil that could inhibit bacterial growth was $1.562 \mathrm{mg} / \mathrm{mL}$ for three bacteria of Enterococcus faecalis, Streptococcus sanguis, and Actinomyces viscose. However, this concentration was $0.39 \mathrm{mg} / \mathrm{mL}$ for Eikenella corrodens. These results show that Satureja essential oil has a better inhibitory effect on the growth of Eikenella corrodens than Enterococcus faecalis, Streptococcus sanguis, and Actinomyces viscose.

Table 1. Median inhibitory concentration and median lethal concentration of Satureja essential oil

\begin{tabular}{ccc}
\hline Microorganism & Median Inhibitory Concentration & Median Killer Concentration \\
\hline Enterococcus faecalis & 562.1 & 562.1 \\
\hline Streptococcus sanguis & 562.1 & 562.1 \\
\hline Eikenella corrodens & 39.0 & 781.0 \\
\hline Actinomyces viscose & 562.1 & 562.1 \\
\hline & & S. Jurnal of \\
\hline
\end{tabular}


Overall, the results of this study showed that the essential oil of Satureja has antibacterial activity and can inhibit the growth of a group of periopathogens, including Enterococcus faecalis, Streptococcus sanguis, Eikenella corrodens, and Actinomyces viscose. It can also inhibit the growth of the mentioned bacteria at a concentration of $1.562 \mathrm{mg} / \mathrm{mL}$ and more Satureja essential oil. It also has an inhibitory effect on the biofilm formation of the mentioned bacteria so that at concentrations of $12.5 \mathrm{mg} / \mathrm{mL}$ and higher of Satureja essential oil, none of the studied bacteria was able to form a biofilm. Therefore, Satureja, as a natural and effective antibacterial, can have a potential role in reducing the probability of incidence and severity of periodontal diseases.

\section{Ethical Considerations}

\section{Compliance with ethical guidelines}

The Ethics Committee of the Arak University of Medical Sciences approved this study (Code: IR.ARAKMU. REC.1397.67).

\section{Funding}

This research did not receive any specific grant from funding agencies in the public, commercial, or not-forprofit sectors.

\section{Authors' contributions}

All authors met the standards of writing criteria based on guidelines of the International Committee of Medical Journal Publishers (ICMJE).

\section{Conflicts of interest}

The authors declare no conflicts of interest.

\section{Acknowledgements}

The authors would like to thank the authorities of Arak University of Medical Sciences for their cooperation in conducting this research. 


\title{
فعاليت ضدباكتريايى و خصوصيات ضدبيوفيلمى اسانس كياه مرزه بر باكترىهاى دخيل در بيمارىهاى بيريودنتال فيرئ
}

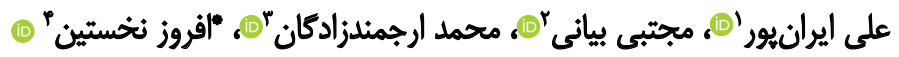

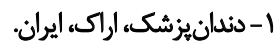

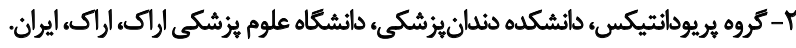

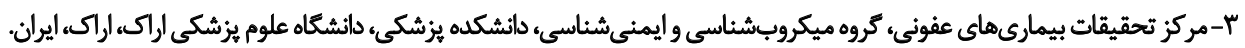

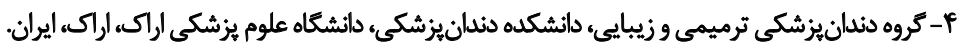

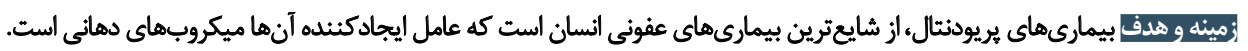

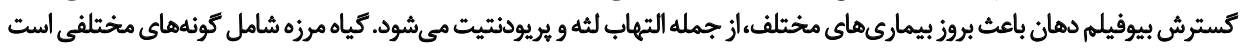

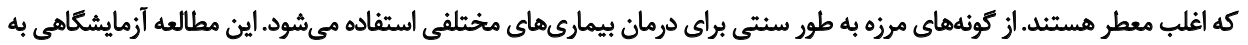

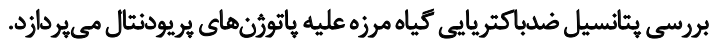

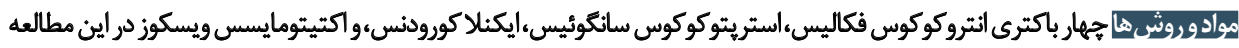

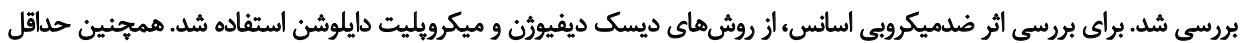

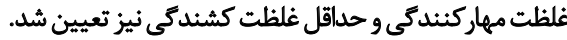

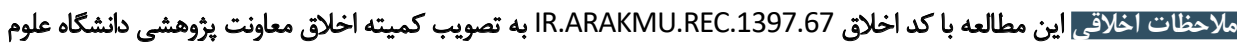

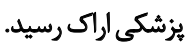

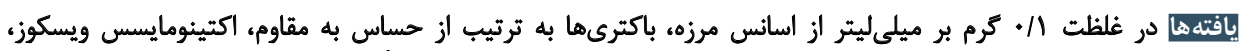

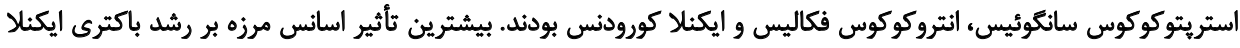

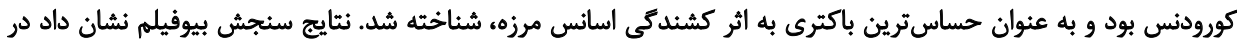

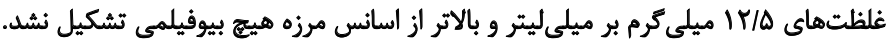

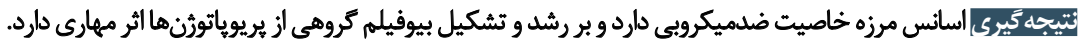

بيوفيلم يك توده متراكم غيركلسيفيه و تركيبشده بانيا

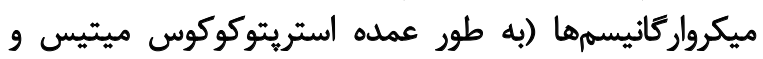

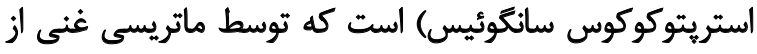

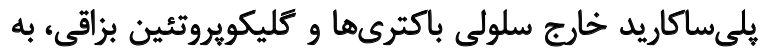
صورتى پايدار و محكم به دندان و ديكر سطوح سخت حفره دهان

1. Enterococcus Faecalis

2. Streptococcus Sanguinis

3. Eikenella Corrodens

4. Actinomyces Viscosus
يوسيدگى دندان و بيمارى هاى يريودنتال از شايعترين بيمارى إنهاى

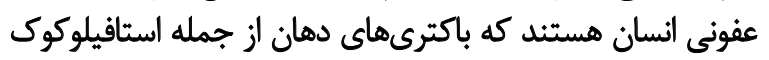

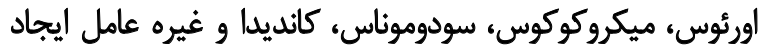

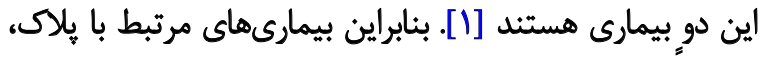

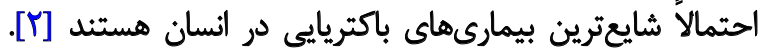

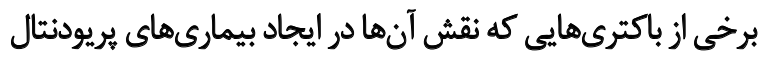

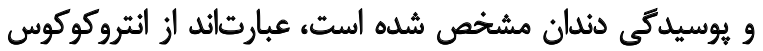

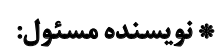

$$
\begin{aligned}
& \text { دانتر افروز نخستين }
\end{aligned}
$$

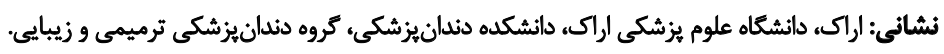

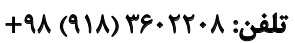

$$
\begin{aligned}
& \text { aست الكترونيكى: afr_na_sa@yahoo.com }
\end{aligned}
$$


از آنجا كه محيط دهان، حاوى گونههاى باكتريال متعددى دري

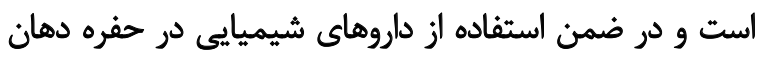

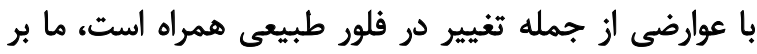

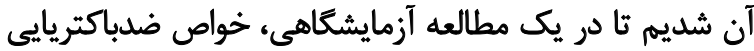

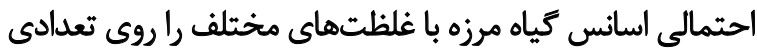

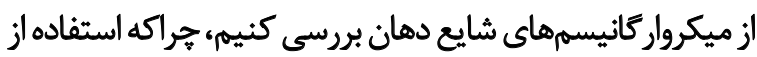

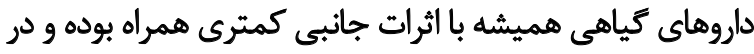

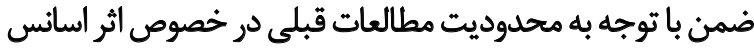

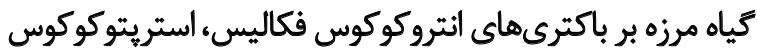

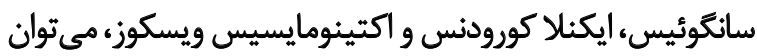

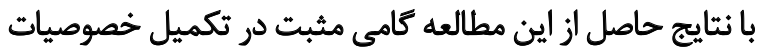
ضدباكتريايى اين كياه برداشت إين مطالعه

\section{مواد و روشها}

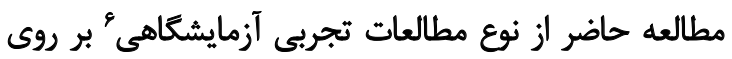

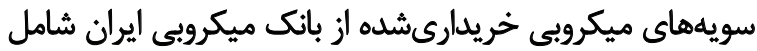

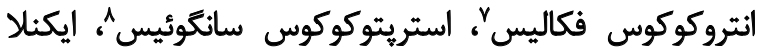

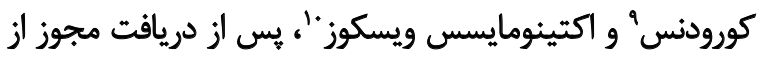

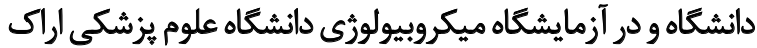

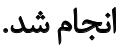

كياه مرزه به صورت تازه از مركز تحقيقات كياهان دارويى

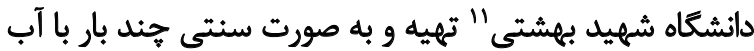

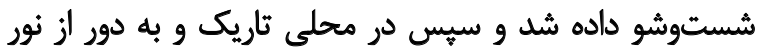

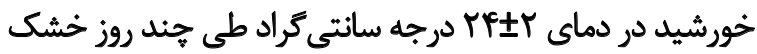

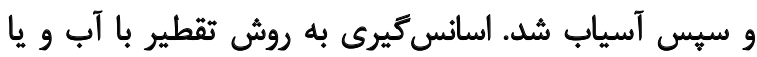

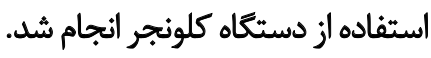

براى انجام آزمون حساسيت ميكروبى به اسانس، با روش

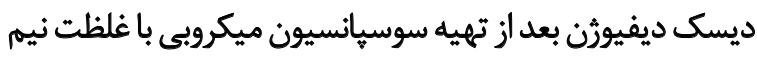

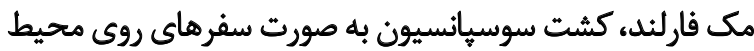

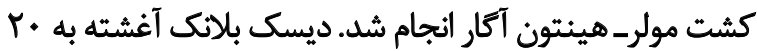

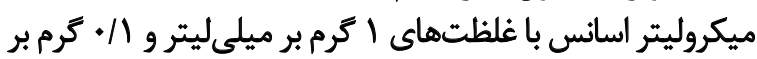

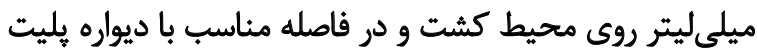

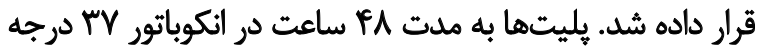

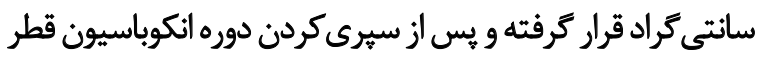

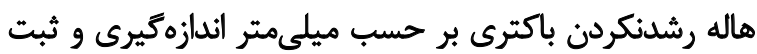

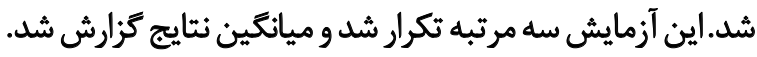
براى تعيين حساسيت ميكروبى به روش ميكرويليت دايلوشن

\footnotetext{
6. Field study

7. PTCC1774

8. PTCC 1449

9. PTCC1391

10. PTCC1202

11. Voucher NO: TARI 83144
}

متصل مىشود [V]]. تسترش بيوفيلم باعث بروز بيمارىهاى

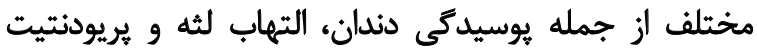

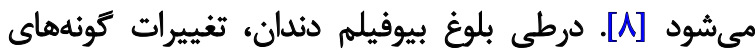

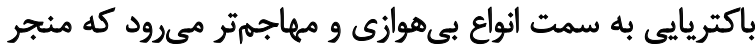

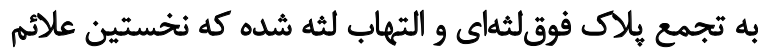

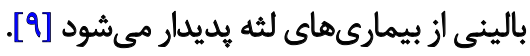

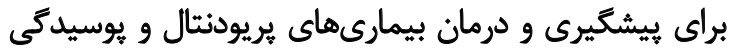

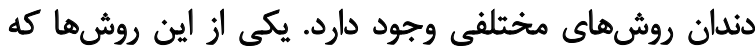

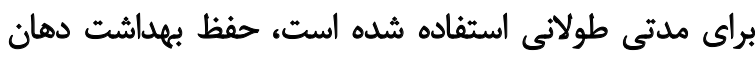

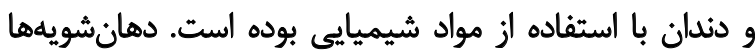

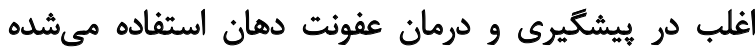

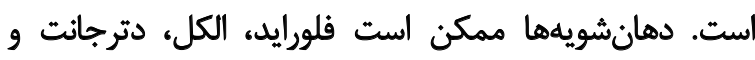

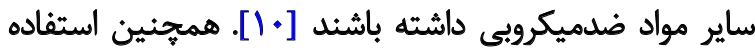

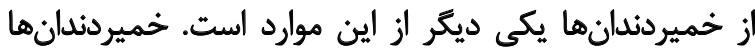

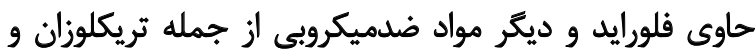

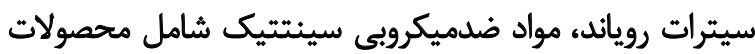

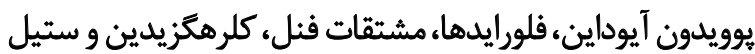

يبريدينيوم هستيند [11].

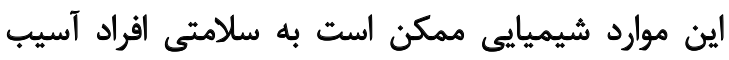

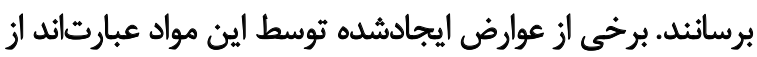

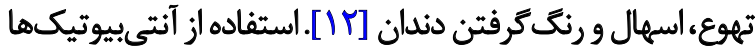

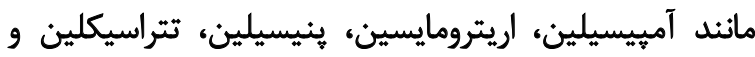

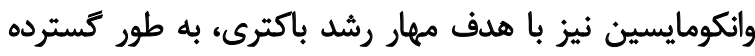

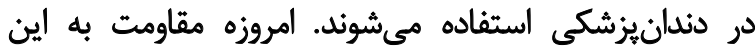

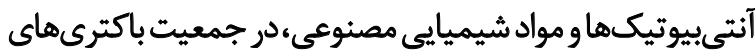

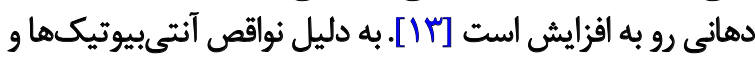

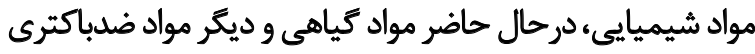

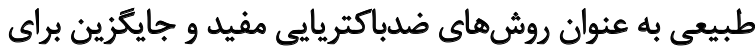

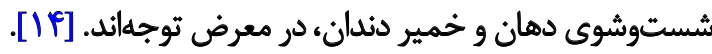

\section{igols}

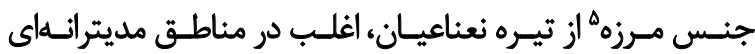

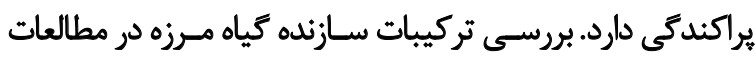

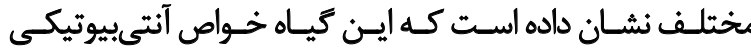

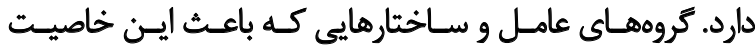

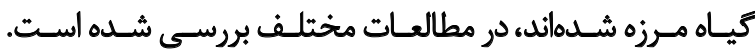

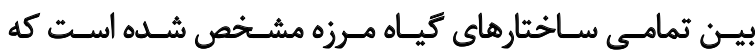

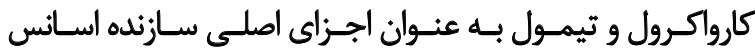

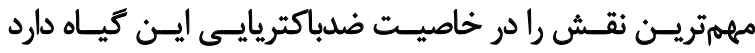

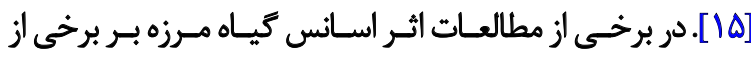

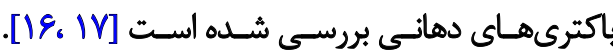




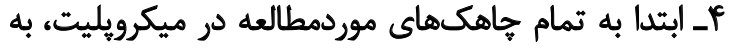

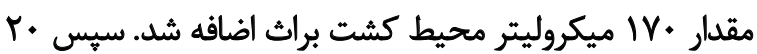

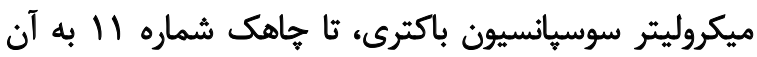

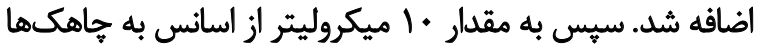

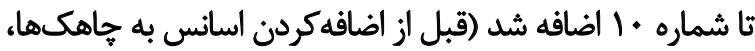

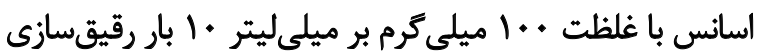

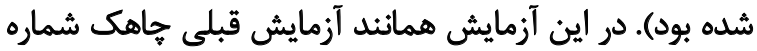

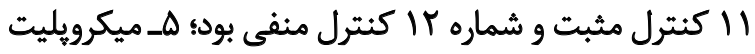

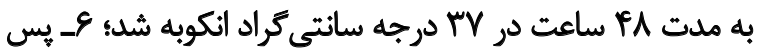

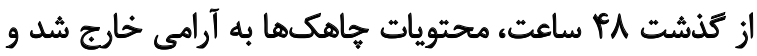

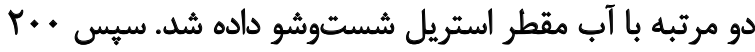

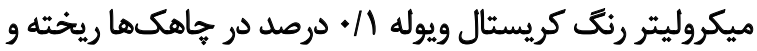

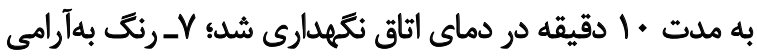

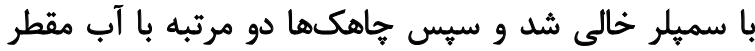

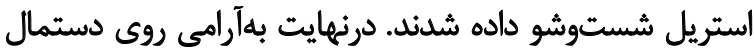

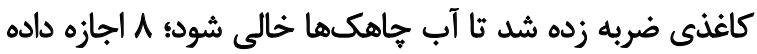

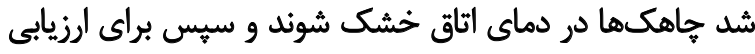

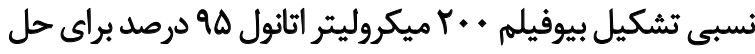

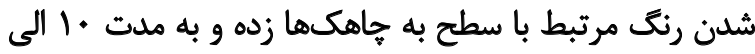

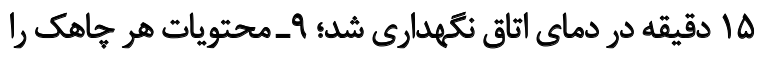

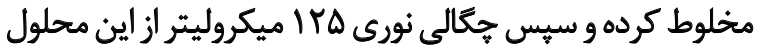

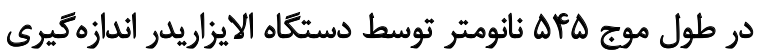

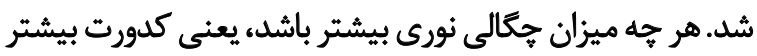

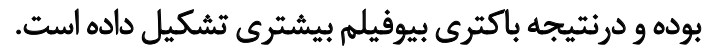

يافتهها

قطر هالههاي رشدنكردن مربوط به غلظتهاي مختلف اسانس

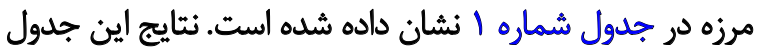

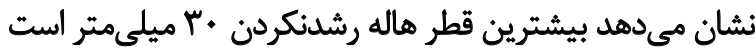

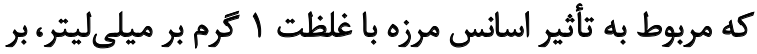

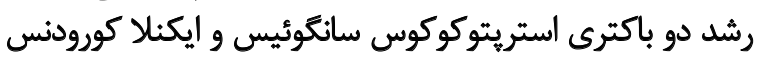

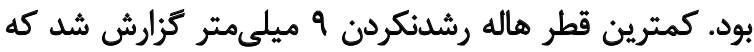

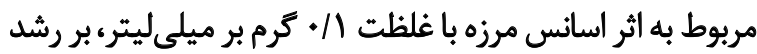

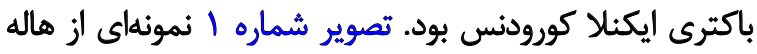

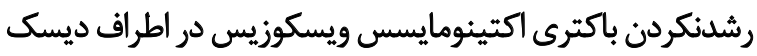
ديفيوزن با غلظت اكرم بر ميلى ليتر اسانس كياه مرزه است. همجنين مقادير حداقل غلظت مهاركنيندكى و حداقل غلظت

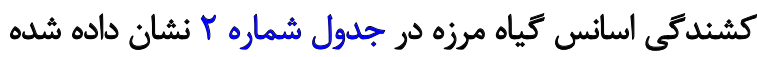

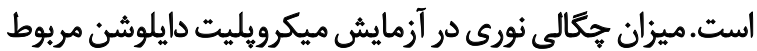

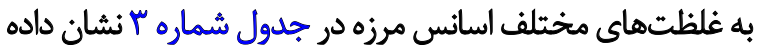

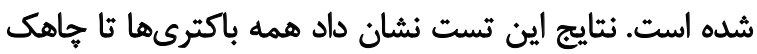

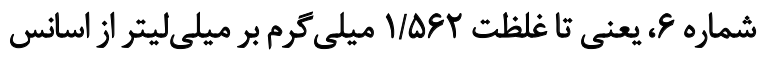

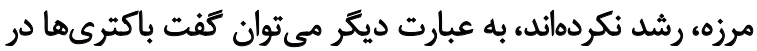

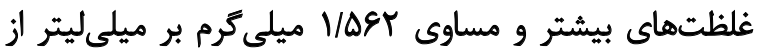

ابتدا از باكترىهايى كه از قبل كشت داده شده بودند و از زمان

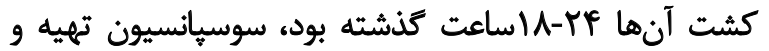

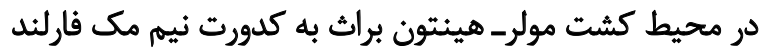

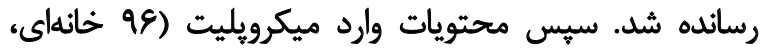

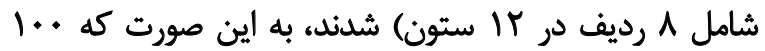

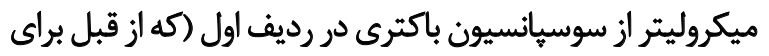

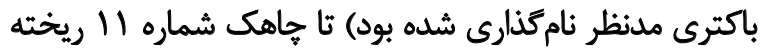

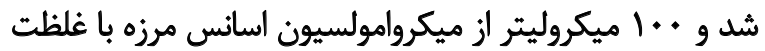

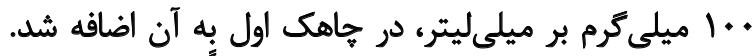

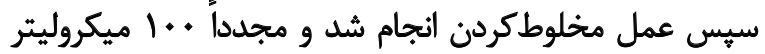

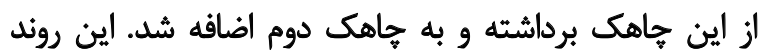

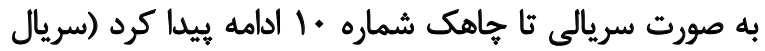

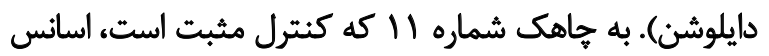

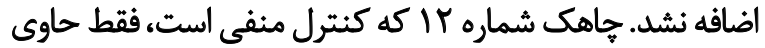

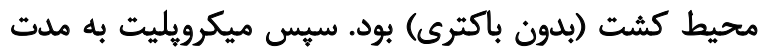

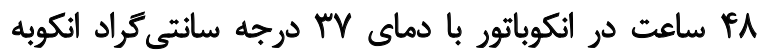

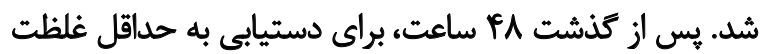

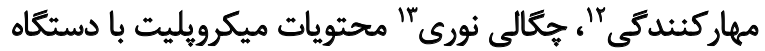

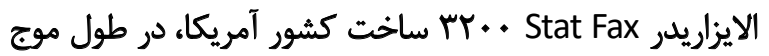

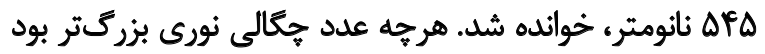

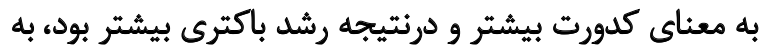

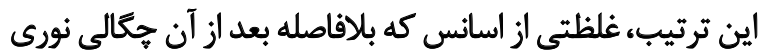

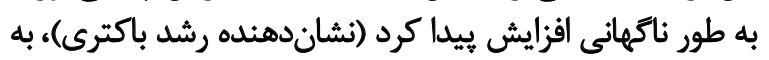

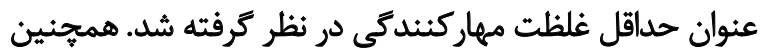

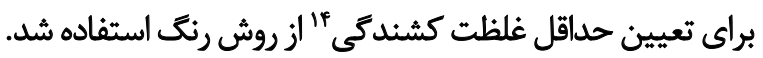

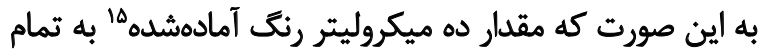

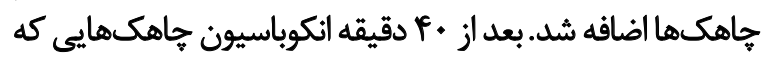

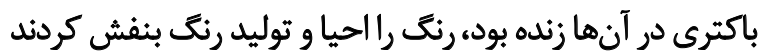

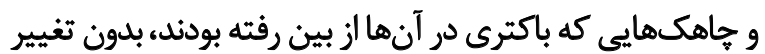

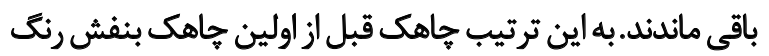
حداقل غلظت كشندكى بود. براى تست سنجش مهار تشكيل بيوفيلم بررسى تشكيل و قدرت بيدي

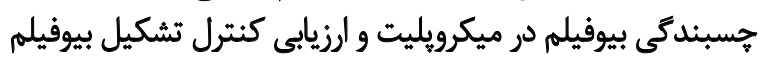

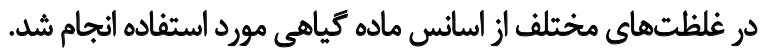

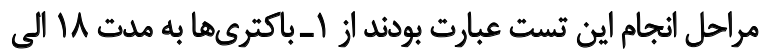

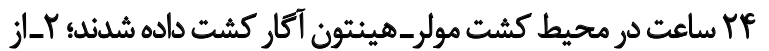

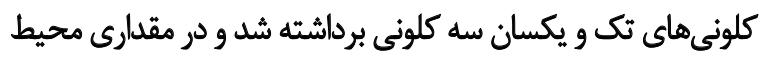

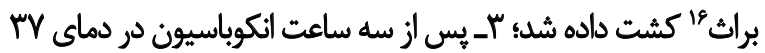
درجه سانتى كراد، كدورت سوسيانسيون به نيم مك فارلند راند رسانده شد؛

12. Minimum inhibitory concentration

13. Optical density

14. Minimum bactericidal concentration

15. Tetrazolium-based

16. Brain heart infusion broth 
جدول ا. قطر هالههاي رشدئكردن بر حسب ميلىمتر مربوط به غلظتهاي مختلف اسانس مرزه

\begin{tabular}{|c|c|c|}
\hline غلظت ا كرم بر ميلي ليتر & غلظت | | كرم بر ميلى ليتر & نام ميكروار كانيسم \\
\hline rf & ir & أتتروكوكوس فكاليس \\
\hline$r$ & 10 & استريتوكوكوس سانكوئيس \\
\hline$r$ & $q$ & ايكنلا كورودنس \\
\hline rA & M & اكتينومايسس ويسكوز \\
\hline
\end{tabular}

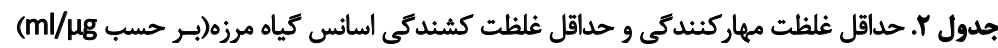

\begin{tabular}{|c|c|c|}
\hline حداقل غلظت كشندكى & حداقل غلظت مهاركثندكى & نام ميكرواروكانيسم \\
\hline V/DET & V/DET & انتروكوكوس فكاليس \\
\hline V/DST & V/DST & استريتوكوكوس سانكوئيس \\
\hline$+/ Y A \mid$ &.$/ 29$ & ايكنلا كورودنس \\
\hline VAST & VAST & اكتينومايسس ويسكوز \\
\hline
\end{tabular}

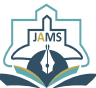

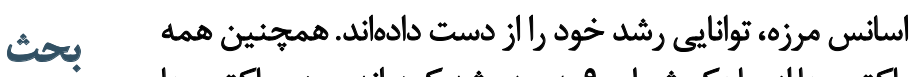

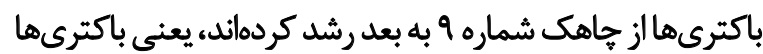

برؤهش حاضر براي بررسى فعاليت ضدباكتريايى اسانس كياه

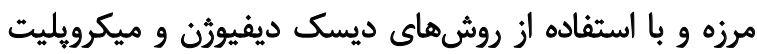

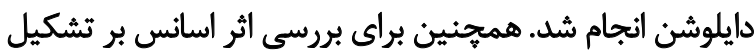

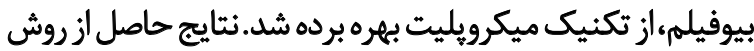

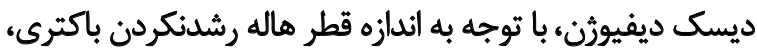

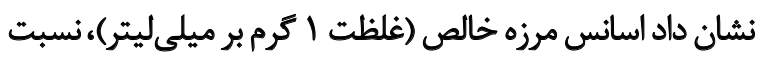

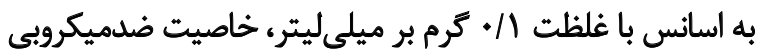

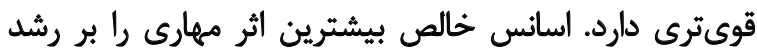

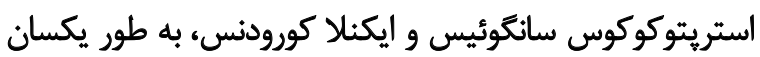

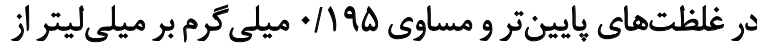
اسانس مرزه، همجنان توانايى رشد خود را حفظ كرئ كردهاند.

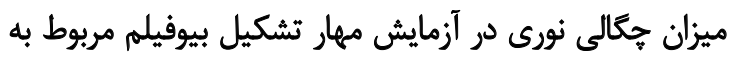

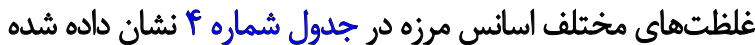

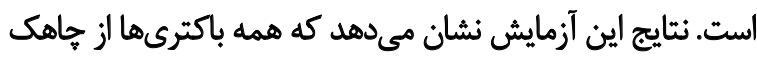

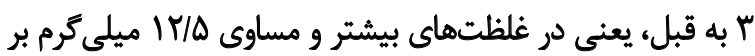

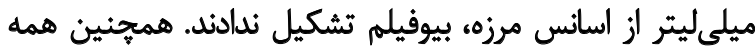

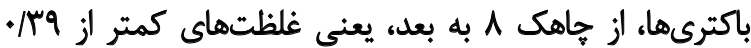

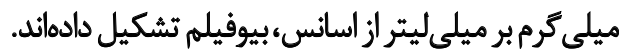

جدول rا. ميزان جكالى نورى در آزمايش ميكرويليت دايلوشن مربوط به غلظتهاى مختلف اسائس مرزه

\begin{tabular}{|c|c|c|c|c|c|c|c|c|c|c|c|c|}
\hline \multicolumn{12}{|c|}{ غلظتهاى مخثلف مرزه بر حسب ميلى مرم بر ميلىليتر } & \multirow[b]{2}{*}{ ميكرواركّانيسم } \\
\hline شنفى & هاهديت & $+1+97$ & +1190 & - /rq & $*|Y \wedge|$ & I/AEY & r/Tro & g/To & $1 T / 0$ & ro & $\Delta$ & \\
\hline$+1+10$ & - &.$/ M m$ & . MEA & $\cdot / m+$ & •/ I &.$M H$ &.$/ 1 T$ &.$/ 118$ & $+M$ &.$/ 110$ &.$/ 11$ & انتروكوكوس فكاليس \\
\hline $.1 \cdot w$ & . pere & . /TAD &.$/ \pi t$ &.$/ 185$ & ./NF. &.$/ 11$ & . /ITA &.$/ 110$ &.$/ I T$ & $\cdot / \cdot V$ & .11 .1 & سانكيوئيس \\
\hline .1 .90 & . MPA &.$/ M T$ & $\cdot r \cdot 1$ &.$/ 18 Y$ &.$/ 148$ &.$M r$ & .1118 &.$/ 11$ &.$/ 111$ &.$/ r$. & .1110 & ايكنلا كورودئس \\
\hline 1.9 & ./MTA &.$/ r n$ & . TMQP & . &.$/ r r r$ &.$/ 111$ & $.11 \cdot 8$ & $\cdot 11 \cdot 1$ &.$/ 11$ & $. / 1+1$ & .1 .94 & اكتينومايسس ويسكوز \\
\hline
\end{tabular}


جدول F. ميزان جمَالى نورى در آزمايش مهار تشكيل بيوفيلم مربوط به غلظتهاى مختلف اسانس مرزه

\begin{tabular}{|c|c|c|c|c|c|c|c|c|c|c|c|c|}
\hline \multicolumn{12}{|c|}{ غلظتهاى مختلف مرزه بر حسب ميلى كرم بر ميلى ليتر } & \multirow[b]{2}{*}{ 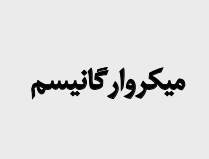 } \\
\hline شنفى & شُشاهد & $+1+98$ & +1190 & म/4q & $+(\mathrm{VA})$ & l/DQY & r/ITo & \&/T৯ & $1 T / \Delta$ & Yo & Q. & \\
\hline $.1 . \Delta f$ & $1 / M$ & I/T & $V / * r$ & $+/ \Lambda \Delta E$ &.$/ 9 \Delta \Delta$ & . /qAT & $+/ 1190$ & . /AAT &.$/ N$ & +11.1 & $\cdot 11+1$ & انتروكوكوس فكاليس \\
\hline $.1 \cdot \Delta Y$ & $V \cdot M$ & $1 / \cdot \Delta$ & $1 / \cdot \Delta A$ &.$/ 1 r$ &.$/ 1 \% 9$ &.$/ 1 F$ &.$/ M Y A$ &.$/ 11 Y$ &.$/ \cdot N \Delta$ & .1 .94 & . AAr & ستريتوئيس \\
\hline $.1+1$ & V/Fer & $V \cdot m$ &.$/ 999$ & $1 /+4$ &.$/ 41$ & ./Arq &.$/ M I r$ & .1 .81 & $\cdot \Lambda \cdot r$ &.$/ * A V$ & .1 .9 & ايكنلا كورودنس \\
\hline .1 .91 & VTror & $M M$ & $1 / * 9$ & . & $\cdot 11 \cdot r$ & .1 .94 & .1 .94 &.$ハ$ &.$/ 114$ & $.1 * 19$ & $1 . \sqrt{9}$ & اكتينومايسس ويسكوز \\
\hline
\end{tabular}

ميلي ليتر به دست آمد. در صورتى كه اين غلظت براي باكترى

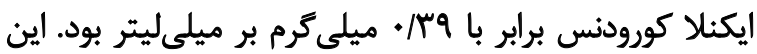

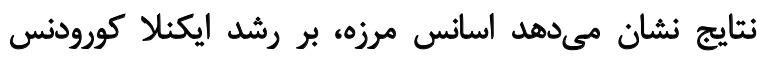
نسبت به انتروكوكوس فكاليس، استريتوكوكوس سانكوئيس

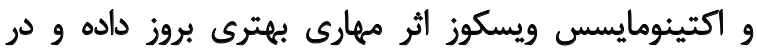

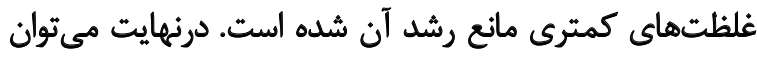

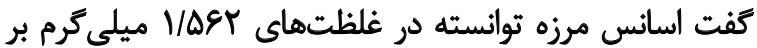
ميلى ليتر و بيشتر، مانع از رشد تمام باكترىهاى مذانى مذكور شود.

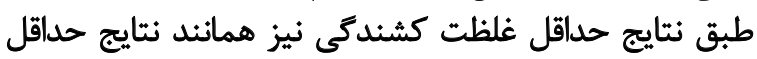
غلظت مهاركنيدكى، حساسترين باكترى نسبت به سيه بـ باكترى

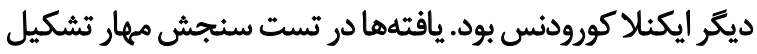

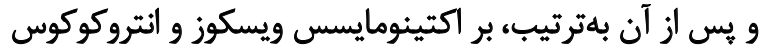

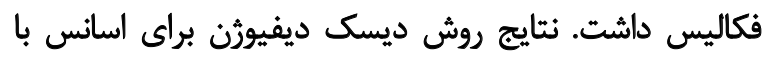

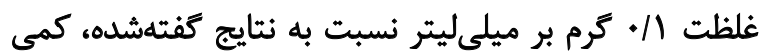

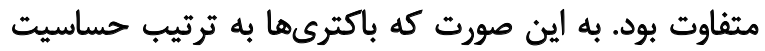
به اسانس، اكتينومايسس ويسكوز، استريتوكوكوس سانكوئيس، انتروكوكوس فكاليس و ايكنلا كورودنس بودند. با بررسي نتايج بهدستآمده از آزمايش ميكرويليت دايلوشن،

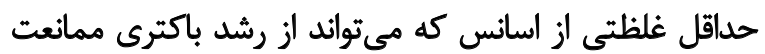

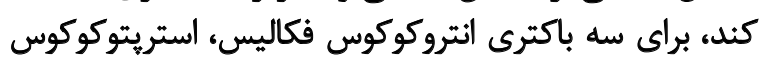

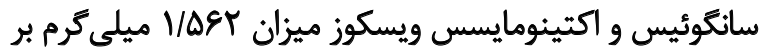

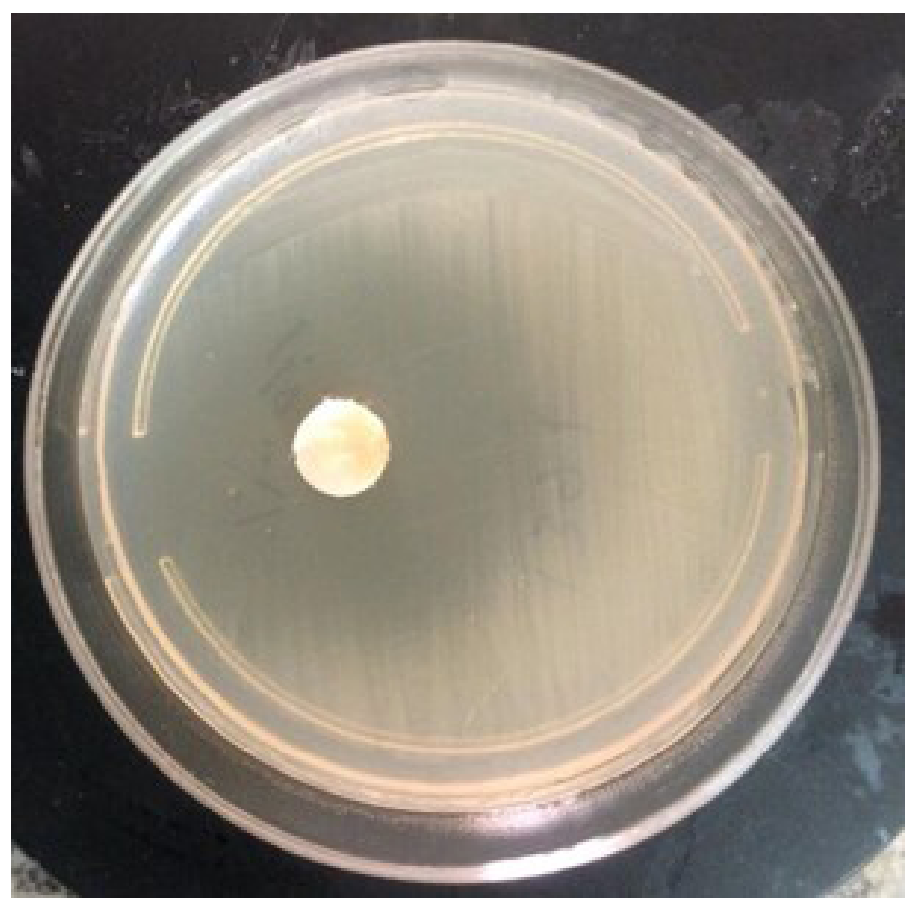

تصوير ا. هاله رشدنكردن باكترى اكتينومايسس ويسكوزيس در اطراف ديسك ديفيوزن با غلظت اكرم بر ميلي ليتر اسانس كياه مرزه 
حساسيت به اسانس در يك سطح قرار داشتند. از اسانسهاى

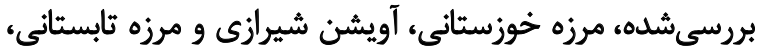

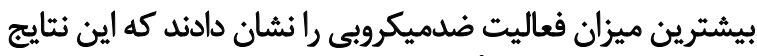
يافتهاى مطالعه ما را تأييد مي كندين

در مطالعه ديكرى اثرات ضدميكروبى اسانس اكالييتوس،

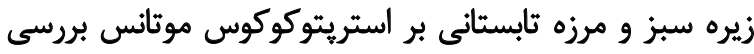

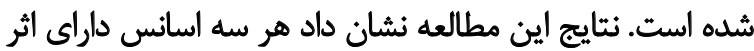

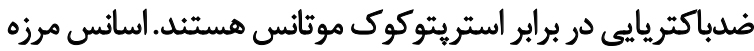

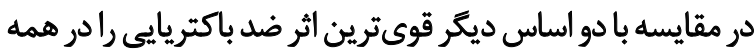

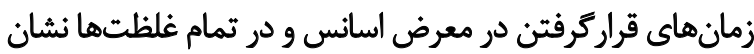

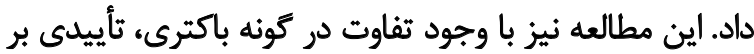

يافتههاى مطالعه ماست [1/1)].

در مطالعه ديكرى كه كرمانشاه و همكاران انجام دادند.

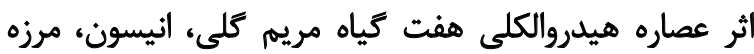

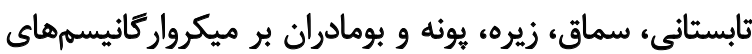

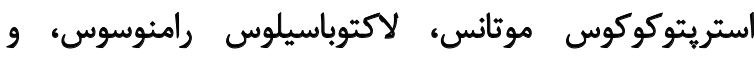

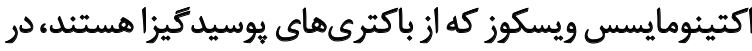

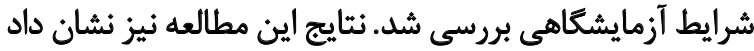

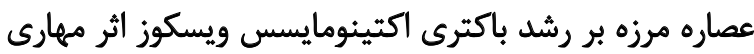

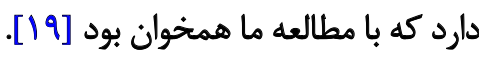

\section{نتيجليَيرى}

نتايج اين مطالعه نشان داد اسانس كياه مرزه خاصيت

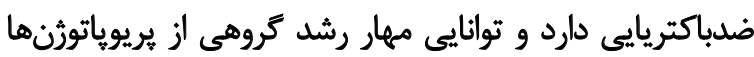

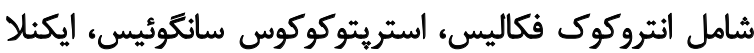

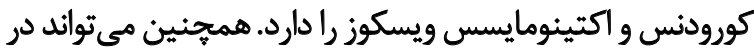

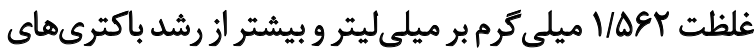

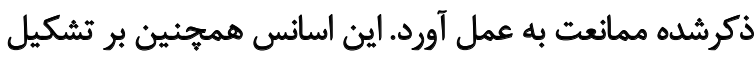

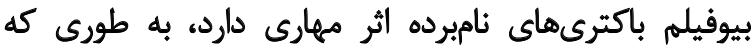

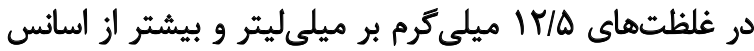

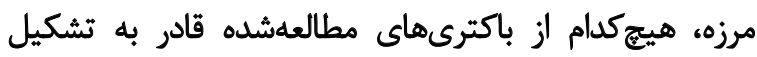

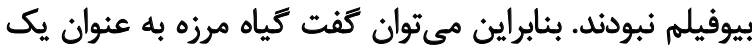

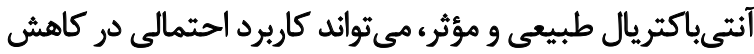
بروز و شدت بيمارى هاى بريودنتال داشته باشدي.

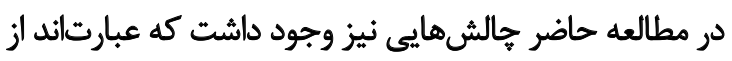

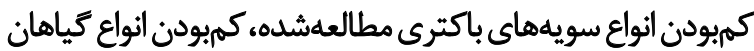

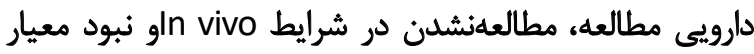

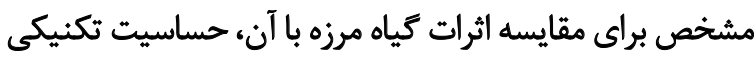

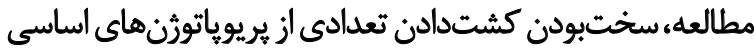

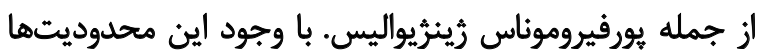

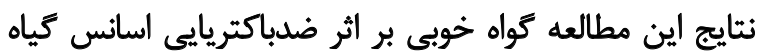

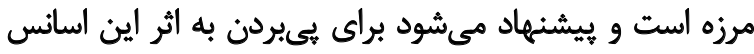

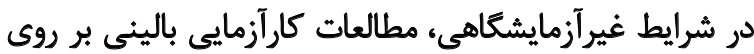

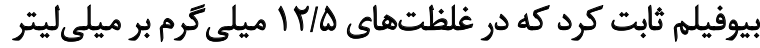

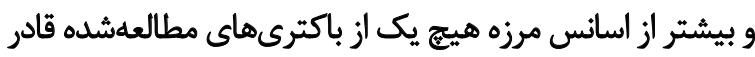

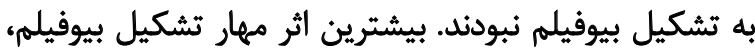

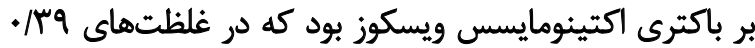
ميلى

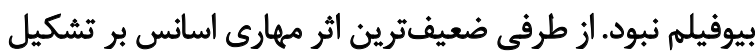

$$
\text { بيوفيلم باكترى انتروكوكوس فكاليس بودين }
$$

نتايج مطالعه حاضر با برخى از يافتههاي ساير مطالعات

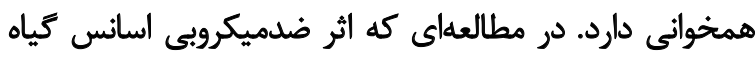

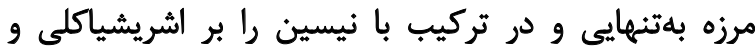

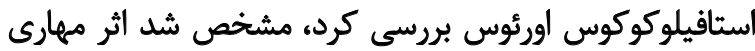

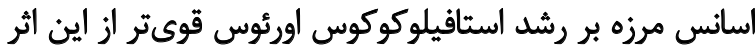

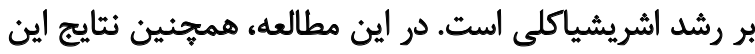

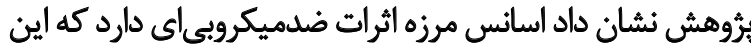

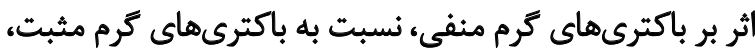

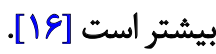

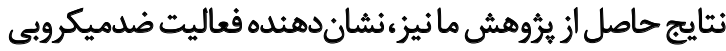

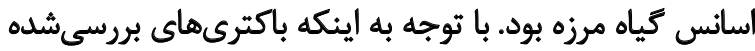

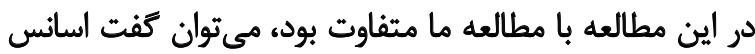

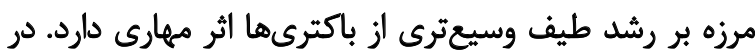

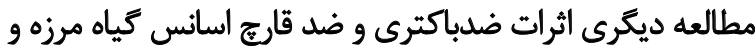

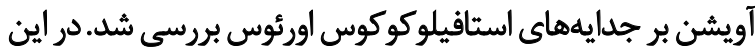

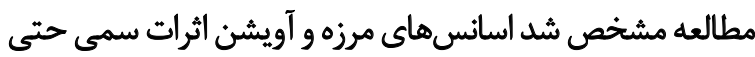

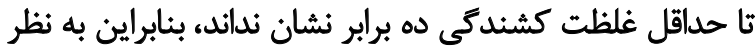

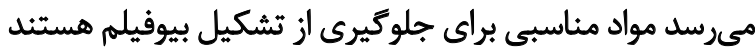

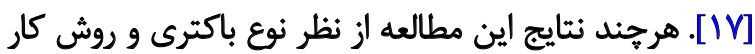

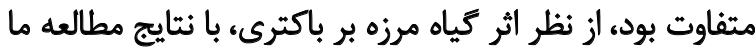

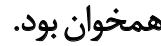

در مطالعه ديكرى كه زمرديان و همكاران انجام دادند،

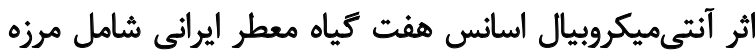

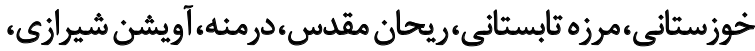

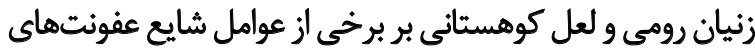

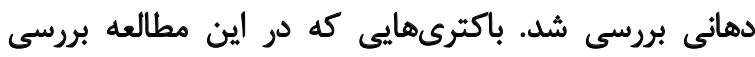

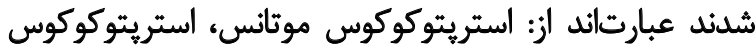

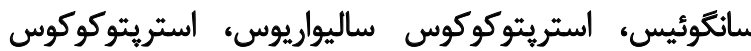

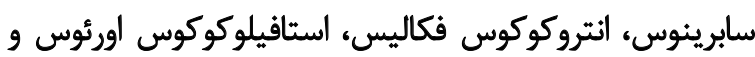

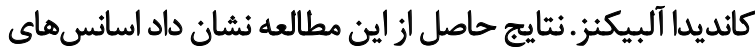

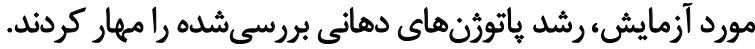

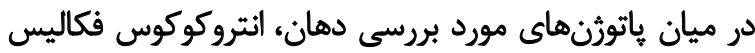

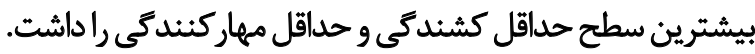
به عبارت ديكر اين باكترى مقاومترين سويه دئ در برابن برابر اسانس

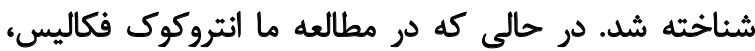
استريتوكوكوس سانكوئيس و اكتينو در مطايسه ما انتروكي ويسكوز از نظر 


$$
\text { نمونههاى انسانى انجام شود. }
$$

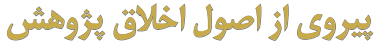

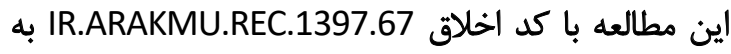
اراك رسيد. كميته اخلاق معاونت يرونشى اخلاف دانشكاه علوم يزشكى

$$
\text { tho sto }
$$

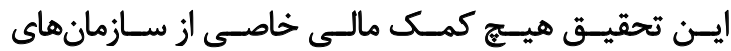

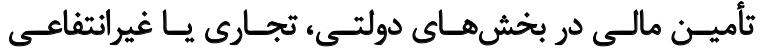

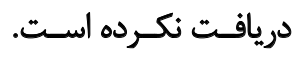

$$
\text { مشاركت نويسندانكان }
$$

تمامى نويسندكان معيارهاى استاندارد نويسندكى بر اساس

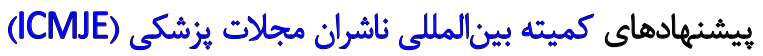

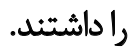

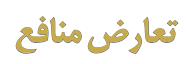

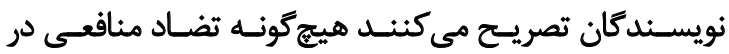

خصـوص يُروهــ حاضـر وجود نــدارد.

$$
\text { و ت }
$$

از همكارىهاى دانشكاه علوم يزشكى اراك در انجام اين

ئروهش تشكر و قدردانى مى دانشود. 


\section{References}

[1] Lasserre JF, Brecx MC, Toma S. Oral microbes, biofilms and their role in periodontal and peri-implant diseases. Mater. 2018; 11(10):pii: E1802. [DOI:10.3390/ma11101802] [PMID] [PMCID]

[2] Murakami S, Mealey BL, Mariotti A, Chapple ILC. Dental plaque-induced gingival conditions. J Periodontol. 2018; 89(Suppl. 1):S17-s27. [DOI:10.1002/JPER.17-0095] [PMID]

[3] Komiyama EY, Lepesqueur LS, Yassuda CG, Samaranayake LP, Parahitiyawa NB, Balducci I, et al. Enterococcus species in the oral cavity: Prevalence, virulence factors and antimicrobial susceptibility. PLOS One. 2016; 11(9):e0163001. [DOI:10.1371/journal.pone.0163001] [PMID] [PMCID]

[4] Zhu B, Macleod LC, Kitten T, Xu P. Streptococcus sanguinis biofilm formation \& interaction with oral pathogens. Future Microbiol. 2018; 13:915-32. [DOI:10.2217/fmb-2018-0043] [PMID] [PMCID]

[5] Kim JH, Choi IA. Periodontal pathogens and the association between periodontitis and rheumatoid arthritis in Korean adults. J Periodontal Implant sci. 2018; 48(6):347-59. [DOI:10.5051/jpis.2018.48.6.347] [PMID] [PMCID]

[6] Li J, Li Y, Zhou Y, Wang C, Wu B. Actinomyces and alimentary tract diseases: $A$ review of its biological functions and pathology. Biomed Res Int. 2018; 2018(3820215):1-8. [DOI:10.1155/2018/3820215] [PMID] [PMCID]

[7] Jamal M, Ahmad W, Andleeb S, Jalil F, Imran M, Nawaz MA, et al. Bacterial biofilm and associated infections. J Chin Med Assoc. 2018; 81(1):711. [DOI:10.1016/j.jcma.2017.07.012] [PMID]

[8] Velsko IM, Fellows Yates JA, Aron F, Hagan RW, Frantz LAF, Loe L, et al. Microbial differences between dental plaque and historic dental calculus are related to oral biofilm maturation stage. Microbiome. 2019; 7(1):102. [DOI:10.1186/s40168-019-0717-3] [PMID] [PMCID]

[9] Chinsembu KC. Plants and other natural products used in the management of oral infections and improvement of oral health. Acta Tropica. 2016; 154:6-18. [DOI:10.1016/j.actatropica.2015.10.019] [PMID]

[10] Magaz VR, Llovera BF, Marti M, Garre A. Clinical impact and cosmetic acceptability of chlorhexidine-enriched toothpaste and mouthwash application on periodontal disease: A randomized clinical study. J Contemp Dent Pract. 2018; 19(11):1295-300. [DOI:10.5005/jp-journals-10024-2421] [PMID]

[11] Hosadurga R, Boloor VA, Rao SN, MeghRani N. Effectiveness of two different herbal toothpaste formulations in the reduction of plaque and gingival inflammation in patients with established gingivitis - A randomized controlled trial. J Tradit Complement Med. 2018; 8(1):113-9. [DOI:10.1016/j.jtcme.2017.04.005] [PMID] [PMCID]

[12] Yousefimanesh H, Amin M, Robati M, Goodarzi H, Otoufi M. Comparison of the antibacterial properties of three mouthwashes containing chlorhexidine against oral microbial plaques: An in vitro study. Jundishapur J Microbiol. 2015; 8(2):e17341. [DOI:10.5812/jjm.17341] [PMID] [PMCID]

[13] Kanwar I, Sah AK, Suresh PK. Biofilm-mediated antibiotic-resistant oral bacterial infections: Mechanism and combat strategies. Curr Pharm Des. 2017; 23(14):2084-95. [DOI:10.2174/13816128226661611241545 49] [PMID]

[14] Sadrnia M, Habibi G, Arjomandzadegan M. [Comparison of disk diffusion and micro-dilution broth methods for evaluation of antimicrobial effects of myrtus extract on methicillin-resistant staphylococcus aureus and escherichia coli ESBL (Persian)]. J Arak Uni Med Sci. 2018; 21(3):75-82.
[15] Serrano C, Matos O, Teixeira B, Ramos C, Neng N, Nogueira J, et al. Antioxidant and antimicrobial activity of Satureja montana L. extracts. J Sci Food Agric. 2011; 91(9):1554-60. [DOI:10.1002/jsfa.4347] [PMID]

[16] Babaei S, Moradi A, Hosseinikia M, Zaveleh OM, Amiri Z, Sohrabi N. [Evaluation of the antimicrobial effects of satureja montana essential oil alone and in combination with nisin on escherichia coli and staphylococcus aureus (Persian)]. J Res Med Dent Sci. 2018; 6(2):54-60.

[17] Sharifi A, Mohammadzadeh A, Zahraei Salehi T, Mahmoodi P. Antibacterial, antibiofilm and antiquorum sensing effects of Thymus daenensis and Satureja hortensis essential oils against Staphylococcus aureus isolates. J Appl Microbiol. 2018; 124(2):379-88. [DOI:10.1111/jam.13639] [PMID]

[18] Zomorodian K, Ghadiri P, Saharkhiz MJ, Moein MR, Mehriar P, Bahran $\mathrm{F}$, et al. Antimicrobial activity of seven essential oils from Iranian aromatic plants against common causes of oral infections. Jundishapur J Microbiol. 2015; 8(2):e17766. [DOI:10.5812/jjm.17766] [PMCID]

[19] Kermanshah H, Kamangar S, Arami S, Kamalinegad M, Karimi M, Mirsalehian $A$, et al. The effect of hydro alcoholic extract of seven plants on cariogenic bacteria-an in vitro evaluation. Oral Health Dent Manag. 2014; 13(2):395-401. [PMID] 
This Page Intentionally Left Blank 